\title{
Local Expansion and Optimization for Higher-Order Graph Clustering
}

\author{
Wenhong Ma, Lijun Cai, Tingqin He, Lei Chen, Zehong Cao, and Renfa Li, Senior Member, IEEE
}

\begin{abstract}
Graph clustering aims to identify clusters that feature tighter connections between internal nodes than external nodes. We noted that conventional clustering approaches based on a single vertex or edge cannot meet the requirements of clustering in a higher-order mixed structure formed by multiple nodes in a complex network. Considering the above limitation, we are aware of the fact that a clustering coefficient can measure the degree to which nodes in a graph tend to cluster, even if only a small area of the graph is given. In this study, we introduce a new cluster quality score, i.e., the local motif rate, which can effectively respond to the density of clusters in a higherorder graph. We also propose a motif-based local expansion and optimization algorithm (MLEO) to improve local higher-order graph clustering. This algorithm is a purely local algorithm and can be applied directly to higher-order graphs without conversion to a weighted graph, thus avoiding distortion of the transform. In addition, we propose a new seed-processing strategy in a higher-order graph. The experimental results show that our proposed strategy can achieve better performance than the existing approaches when using a quadrangle as the motif in the LFR network and the value of the mixing parameter $\mu$ exceeds 0.6.
\end{abstract}

Index Terms-community detection, community search, higher-order graph clustering, hypergraph clustering, motif clustering.

\section{INTRODUCTION}

\section{A. Background}

In the past decade, graph clustering has become a popular topic in analyzing the structure of graphs [1]. In a graph or network, clusters are typically groups of vertices with a higher probability of connecting to each other than to members of other groups, although other patterns are possible [2]. Clusters have many application scenarios in the Internet of Things [3], including sensor networks [4], vehicular ad hoc networks [5][7], and in-vehicle networks [8]-[10]. Community detection can be viewed as a problem of graph clustering in which each community corresponds to a cluster in the graph [11], [12]. In this study, the two notions are interchangeable. Community

This work was supported by the National Natural Science Foundation of China $(61472127,61272395)$, China Postdoctoral Science Foundation (2013M540628, 2014T70767), Natural Science Foundation of Hunan Province (14JJ3107), and Excellent Youth Scholars Project of Hunan Province (15B087). (corresponding author: Lijun Cai).

Wenhong Ma, Lijun Cai, Tingqin He, and Renfa Li are with the College of Information Science and Engineering, Hunan University, Chansha, China (email: wenhongma, ljcai, hetingqin, lirenfa@ hnu.edu.cn)

Lei Chen is with the School of Information and Electrical Engineering, Hunan University of Science and Technology, Xiangtan, China (email: chenlei@hnust.edu.cn)

Zehong Cao is with the Discipline of ICT, School of Technology, Environments and Design, College of Sciences and Engineering, University of Tasmania, TAS, Australia (email: zehong.cao@utas.edu.au) detection algorithms are generally divided into global-based and local-based community detection [13]-[15]. Global-based community detection requires knowledge of the entire network structure, and current networks are too large to change in real time, such as the World Wide Web [14]. In contrast with global-based community detection, local-based community detection requires knowledge of only a small area of the relevant graph of the input node. Consequently, these approaches are more adaptable to large-scale and real-time changes in a network and haves a more extensive development space [16]. The input node is known as a seed, and a community search is a common application scenario in which a given seed finds its community. Seeds are typically processed to improve the community quality before the community is identified [17].

Traditional community detection is based on a single vertex or edge, and it disregards the interactions among vertices, which are likely to capture social phenomena. These interactions correspond to induced subgraphs of networks that contain multiple vertices and edges and represent the information from different interactions among multiple vertices, and this kind of subgraph is also refered to as a motif [18]. The motif of a network is crucial to organization of complex networks [19], [20] and has a wide range of application scenarios in many fields, such as carbon exchange models in food chains, resource allocation in the Internet of Things [7], and analysis of small structures in social networks [21]. The use of motifs as atomic units in graph clustering is known as higher-order graph clustering. Similar to traditional graph clustering, higher-order graph clustering is used to identify clusters with tighter connections between internal units than between external units. If the unit is a node, then it is a graph cluster in the typical sense. Triangles are social and biological network motifs that play important roles [18], [21], [22]. In the current study, we use triangles as the main motifs, but to increase generality, we also use undirected quadrilaterals as motifs (Figure 1).
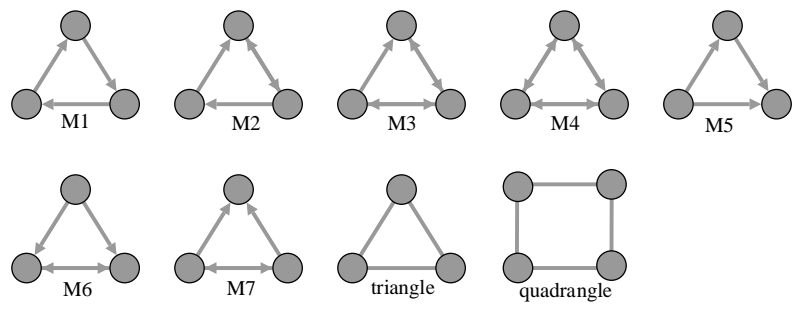

Fig. 1. Example of motif $\mathrm{M}$. 


\section{B. Motivation}

Benson et al. [23] proposed motif conductance based on conductance, a cluster quality scoring method, designed to incorporate higher-order structure and handle edge directions. However, if no motif exists in the cluster, the motif conductance cannot accurately reflect the quality of the cluster, as shown in Figure 2B. We introduce a new cluster quality score, namely, the local motif rate, which calculates the density of the motif in a cluster. This score can be used to control the cluster size by adjusting parameter $\alpha$. Given a graph $G$ and a motif $M$, and under the same parameter $\alpha$, if the number of motifs whose nodes are all in cluster $C$ is considerably higher than that of motifs where only a portion of nodes are in cluster $C$, then cluster $C$ has a good (high) score for motif $M$. Figure 2A illustrates the concept of the local motif rate, where the number of $\mathrm{M}$ in $C$ is counted, but not the edges. In this manner, edges that are not in a given motif do not contribute to the local motif rate.

Higher-order graph clustering can also be classified into global and local methods. Benson et al. [23], [24] recently used spectral clustering to complete higher-order graph clustering. Because this algorithm is based on a global algorithm and is not suitable for large-scale real-time changing networks, Yin et al. [12] proposed the motif-based approximate personalized PageRank (MAPPR) algorithm, which sorts the motif conductance after applying approximate personalized PageRank (APPR) [25]. The MAPPR considers graph clustering as a graph partitioning problem. After calculating the number of occurrences of all nodes in the motif for the entire graph, the original graph is transformed into a matrix of motif counts. Each given seed is required to traverse the entire graph that contains the motif and the graph is divided into two portions: cluster $C$ and other parts, i.e., it is still required to know the structure of the entire graph to count the motifs and convert them to a matrix. Thus, MAPPR is not a purely local method. In this study, we develop the motif-based local expansion and optimization (MLEO) algorithm, a purely local method, which is only required to know the nodes associated with the seed node instead of the entire network. In addition, Benson et al. [23] and Yin et al. [12] converted the higher-order graph into a standard weighted graph and used spectral clustering or APPR algorithms on this weighted graph, but this conversion can cause performance distortion [26]. Our algorithm is based on the concept of local expansion and optimization [16], [27][29], and achieves good performance on higher-order graphs without transforming it into a weighted graph. This algorithm can be applied to hierarchical and overlapping community detection.

The seed strategy is essential in community detection and community search [13]. In community detection, seeds typically are selected from important nodes, and the community gained from these important nodes is usually meaningful. Use of node degrees to represent the node importance is a common method. In community search, although the seed is a given node, the processing of seeds is crucial to improve the quality of a community [17]. The traditional seed processing strategy simply takes a given node and its neighbor nodes as the initial structure, and cannot apply it to higher-order graph clustering. Yin et al. [12] proposed a strategy that uses the local minimum of motif conductance as a seed. However, this method merely focuses on finding better seeds in the entire network and cannot obtain a good initial structure for any given node. Therefore it cannot effectively work on community search. We propose a new motif-based processing strategy that treats a given node and the motifs with the given node as seeds. We compare several processing strategies in the networks and prove that our method is more adaptive to higher-order graphs.

\section{Our contributions}

In summary, our main contributions are listed as follows.

- A new clustering scoring method known as the called local motif rate is proposed for higher-order graph clustering. This method determines the quality of the cluster by calculating the density of the motif in the cluster and solves the error quality score of motif conductance when no motif exists in the cluster. Simultaneously, this method can also react to cluster quality with different particle sizes of parameter $\alpha$.

- A new algorithm (MLEO) for higher-order graph clustering is proposed based on local expansion and optimization. This is a purely local algorithm that uses a greedy approach to find clusters that maximize quality scores on the higher-order graph, without converting it into a weight graph, to avoid conversion caused performance distortion. This algorithm can be applied to directed, undirected, and overlapping hierarchical networks, and even to a weighted network with a simple adjustment.

- A new seed processing strategy (motif seed) is proposed that improves the final community by obtaining an initial community with better clustering quality scores. Experiments show that this strategy is suitable for higher-order graph clustering.

\section{PRELIMINARY}

We formally describe the higher-order graph clustering problem, and review important concepts in graph clustering. We also introduce a new cluster quality score, namely, the local motif rate.

\section{A. Problem statement}

Given a graph $G$, which might be a directed or an undirected graph, $G=(V, E)$ represents an unweighted graph, and $G=$ $(V, E, W)$ represents a weighted graph. We define $V, E$, and $W$ to represent the node sets, edge sets, and edge weight sets, respectively. Let $v_{i}$ denote node $i, e_{i j}$ denote the edge from $v_{i}$ to $v_{j}$, and $w_{i j}$ denote the weight of node $v_{i}$ to node $v_{j}$. Additionally, $v_{i} \in V, e_{i j} \in E$, and $w_{i j} \in W$. For any set $X$, this study uses $|X|$ to denote its size. The motif $M$ has $k$ nodes. The objective of higher-order graph clustering is to find higher-order clusters $C_{1}, \ldots, C_{P}$ with high quality. In this study, we focus on local higher-order graph clustering, which uses only local information to find high-quality clusters. 


\section{B. Motif conductance}

When the motif is a node, the motif conductance represents conductance in graph theory, which is a measure of connectivity that uses the remainder of the graph to identify highquality clusters, and the score of the cluster is obtained by calculating the cut ratio [15]. Cut and volume are the bases of conductance. We define $\operatorname{links}\left(S_{i}, S_{j}\right)$ as the sum of the edge weights between vertex sets $S_{i}$ and $S_{j}$.

Cut. The cut of a cluster $C$ is defined as the sum of edge weights between $C$ and its complemets $\bar{C}$ :

$$
\operatorname{cut}(C)=\operatorname{links}(C, \bar{C}) \text {. }
$$

Volume. The volume of a cluster $C$ is defined as the sum of the edge weights between $C$ and $V$ :

$$
\operatorname{vol}(C)=\operatorname{links}(C, V) .
$$

Conductance. The conductance of a cluster is defined as the cut divided by the least number of the edges incident on either set $C$ or $\bar{C}$ :

$$
\phi(C)=\frac{\operatorname{cut}(C)}{\min \{\operatorname{vol}(C), \operatorname{vol}(\bar{C})\}} .
$$

By definition, $\phi(C)=\phi(\bar{C})$ and lower values indicate higher cluster quality.

When motif $\mathbf{M}$ has multiple nodes, motif conductance is used to reflect the quality of higher-order clusters. We define $\operatorname{links}_{M}\left(S_{i}, S_{j}\right)$ as the sum of instances of motif $\mathrm{M}$, where there are $r$ nodes in set $S_{i}$ and other nodes in $S_{j}, r \in[1, k-1]$.

Motif cut. The motif cut of a cluster $C$ is the number of instances of $\mathrm{M}$ with at least one end point (i.e., node) in $C$ and at least one end point in $\bar{C}$ :

$$
\operatorname{cut}_{M}(C)=\operatorname{links}_{M}(C, \bar{C}) .
$$

Motif volume. The motif volume of a cluster $C$ is the number of occurrences for nodes in $C$, counted over each node in every instance of $\mathrm{M}$ :

$$
\operatorname{vol}_{M}(C)=\sum_{v_{j} \in C} \operatorname{link} s_{M}\left(\left\{v_{j}\right\}, V\right) .
$$

Motif conductance. The motif conductance of a cluster $C$ is the ratio of $\operatorname{cut}_{M}(C)$ to $\min \left\{\operatorname{vol}_{M}(C), \operatorname{vol}_{M}(\bar{C})\right\}$ :

$$
\phi_{M}(C)=\frac{\operatorname{cut}_{M}(C)}{\min \left\{\operatorname{vol}_{M}(C), \operatorname{vol}_{M}(\bar{C})\right\}} .
$$

Motif conductance is used to reflect the quality of the cluster by minimizing the ratio of the motif cut to the motif volume. As such, $\operatorname{cut}_{M}(C)$ is the number of motifs in cut, which is the unit of motif, and $\operatorname{vol}_{M}(C)$ is the number of times that each node in $C$ appears in motif $M$, i.e., the number of nodes. The counting units of cut and volume are inconsistent, and thus certain errors might exist. We offer two examples of calculating the motif conductance in Figure 2. The dotted region is a cluster. In Figure 2A, motif conductance obtains the correct response cluster quality score. In Figure $2 \mathrm{~B}$, the corresponding motif conductance value is $3 / 5$, and no complete motif is found in cluster $C$. In other words, the motif should have the worst quality score of 1 for cluster $C$.
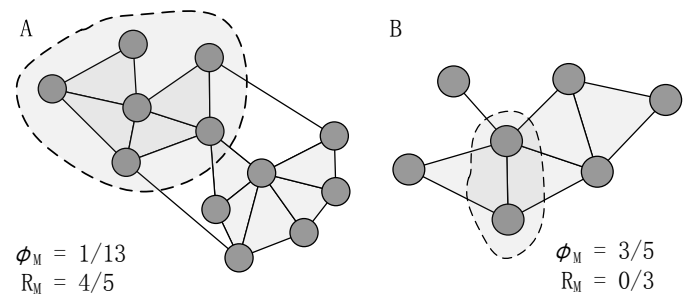

Fig. 2. Steps in calculating motif conductance and local motif rate when motif $\mathrm{M}$ is the triangle and the area enclosed by the dotted line is cluster $\mathrm{C}$. In $\mathrm{A}$, both methods measure the clustering score. In $\mathrm{B}$, the motif conductance score is not equal to 1 , which is the worst score for cluster $\mathrm{C}$ without motif M.

In addition, $\phi_{M}(C)=\phi_{M}(\bar{C}), \bar{C}$ is an unconnected graph, and the obtained $\phi_{M}(\bar{C})$ is meaningless, i.e., it cannot be used as a quality scoring function to reflect the quality of clusters in this situation. The $\operatorname{vol}_{M}(C)$ disregards the atomicity of the motif in the higher-order graph cluster, i.e., higher-order graph clustering uses the motif as an atomic unit rather than a node, which might cause incorrect boundary motifs. Thus, we introduce a new higher-order cluster quality score.

\section{New cluster quality score}

In traditional graph clustering, in addition to conductance, the quality of density metrics is also a method commonly used to reflect cluster quality. This metric is equal to the ratio of the sum of intra-cluster weights to the sum of intra-cluster and inter-cluster weights [2], [14], [30].

Intra-cluster weight. The intra-cluster weight of cluster $C$ is defined as the sum of edge weights in cluster $C$ :

$$
W_{i n}(C)=\operatorname{links}(C, C) \text {. }
$$

Inter-cluster weight. The inter-cluster weigt of cluster $C$ is defined as the sum of edge weights between $C$ and its complements $\bar{C}$ :

$$
W_{\text {out }}(C)=\operatorname{links}(C, \bar{C}) .
$$

Relative density. The relative density of cluster $C$ is defined as the ratio of $W_{i n}(C)$ to the sum of $W_{i n}(C)$ and $W_{\text {out }}(C)$ :

$$
R(C)=\frac{W_{\text {in }}(C)}{W_{\text {in }}(C)+W_{\text {out }}(C)} .
$$

In this study, we introduce a new cluster quality score, local motif rate, which reflects the quality of a cluster by measuring the density of the "atomic unit" motif $\mathrm{M}$ in a higher-order graph.

Number of intra-cluster motif. The number of instances of motif $M$ in which all nodes are in $C$ :

$$
N_{i n}(C)=\operatorname{links}_{M}(C, C) .
$$

Number of inter-cluster motif. The number of instances of motif $M$ in which only some of the nodes are in $C$ :

$$
N_{\text {out }}(C)=\operatorname{link}_{M}(C, \bar{C}) \text {. }
$$

Local motif rate. The local motif rate of motif $M$ of cluster $C$ is defined as the ratio of $N_{\text {in }}(C)$ to $\left(N_{\text {in }}(C)+N_{\text {out }}(C)\right)^{\alpha}$ :

$$
R_{M}(C)=\frac{N_{\text {in }}(C)}{\left(N_{\text {in }}(C)+N_{\text {out }}(C)\right)^{\alpha}} .
$$


Parameter $\alpha=1$ indicates the relative density of the higherorder cluster. Parameter $\alpha$ can be used to adjust cluster size.

Compared with motif conductance, the local motif rate shows the following advantages. 1) The local motif rate addresses the motif of the higher-order graph as the atomic unit and does not count the nodes, thereby avoiding repeated counting when no motif exists in the cluster. 2) The local motif rate can use a different parameter $\alpha$ to adjust cluster size. As $\alpha$ increases, the size of a community gradually decreases. From another perspective, the stability of a cluster can be reflected by the range of $\alpha$, i.e., when the range of $\alpha$ in a cluster is large, then the cluster is stable.

Figure 2 shows two examples of motif conductance and local motif rate calculations. In Figure 2B, when cluster $C$ does not include a motif $M$, the local motif rate is equal to the worst value (0). If $\alpha=1$, then $R_{M}(C) \in[0,1], R_{M}(C)$ represents the density of $C$, and values closer to 1 mean better quality. Hence, higher-order clustering is converted to find a high $R_{M}(C)$ value of the region.

\section{PRoposed ALGORITHM}

Our algorithm uses local expansion and optimization. This approach generally relies on a local benefit function that characterizes the quality of a densely connected subgraph. In this work, we use the local motif rate, and our method differs from the usual non-higher-order local expansion and optimization. Higher-order graph clustering exhibits its own characteristics and is based on motif $\mathrm{M}$ with $\mathrm{k}$ nodes. If $k-1$ nodes are expanded at one time, then too many combinations result, which considerably increases the calculation amount. If only one node is expanded, then the obtained cluster will has poor quality. In accordance with this feature, complexity and accuracy are both considered in expanding and optimizing, and different expansion and optimization methods are proposed.

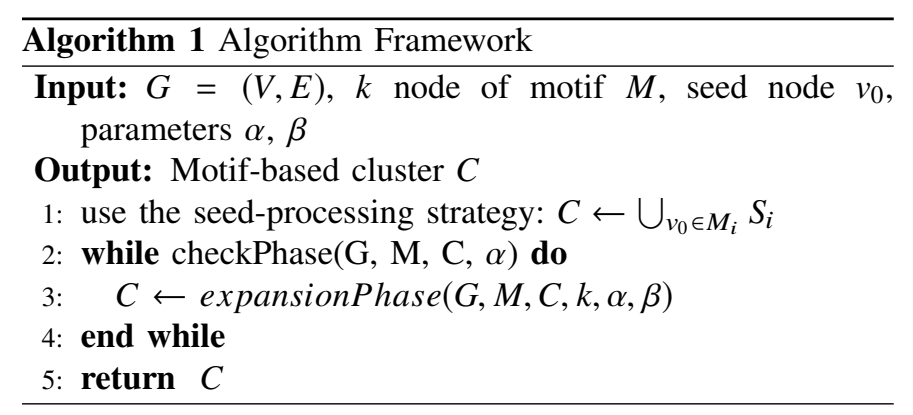

Our algorithm is divided into three components. The first component is the seed phase, which addresses a given node to obtain the initial community. The second component is the expansion phase, which uses a clustering fitness function to expand the nodes to join the community. The third component is the check phase, which checks whether a node is able to reduce the cluster score. If such a node exists, then it is deleted. Algorithm 1 describes the processes of the three phases. The algorithm framework is explained as follows.

1) Line 1, seed phase (Section III-A). The seed strategy is used to process the seed node $v_{0}$, and the initial cluster $C$ is obtained.



Fig. 3. Example of $d_{M}\left(v_{0}\right)$ and motif seed when the motif is a triangle.

2) Line 2, check phase (Section III-C). The initial community $C$ is checked, and the negatively affected nodes are deleted.

3) Line 3, expansion phase (Section III-B). Cluster $C$ is continuously expanded until the maximum cluster quality score is obtained.

In the algorithm, the granularity of a community can be adjusted by changing the parameter $\alpha$. When entering or exiting the expansion phase, a check phase is performed to determine whether deleting the node can improve the local benefit function. Additionally, the checking frequency in the expansion phase is set by parameter $\beta$, which is generally related to the graphical scale. The following subsections describe the steps of each phase in detail.

\section{A. Seed phase}

Seeds are the initial structure of a cluster. In community detection, the center or important node is typically viewed as the seed. A node with a high degree is usually the central node of the network. However, in a higher-order graph, a simple count of all the edges to a node might fail to reflect the importance of a node. In Figure 3, for example, the degrees of nodes $v_{0}$ and $v_{1}$ are 4 and 3 , respectively. However, $\phi_{M}\left(V \backslash v_{0}\right)=\phi_{M}\left(V \backslash v_{1}\right)$ and $R_{M}\left(V \backslash v_{0}\right)=R_{M}\left(V \backslash v_{1}\right)$, indicating that $v_{0}$ is not more important than $v_{1}$ for a higherorder graph, even if the degree of $v_{0}$ is greater that of $v_{1}$. The reason for such a finding is that $e_{07}$ is counted and not involved in the motif. Simply removing the edges that do not participate in the motif is not a good solution. For example, for $v_{2}$ and $v_{3}$ in Figure 3, $\phi_{M}\left(V \backslash v_{2}\right)<\phi_{M}\left(V \backslash v_{3}\right)$ and $R_{M}\left(V \backslash v_{2}\right)>R_{M}\left(V \backslash v_{3}\right)$. Evidently, $v_{2}$ is more suitable as a seed, but the both have equal degree and edge are involved in the motif. We considered motif clustering from the perspective of hypergraph [26], [31]-[33], suggesting that the hyperedges correspond to motifs [26], [33], so it is reasonable to apply the concept of degree from hypergraph in this study. To distinguish from the traditional degree, it is known as the M-degree in this study.

Given a graph $G$ and motif $M$, the node motif M-degree $d_{M}$ is the number of occurrences of a node in motif $M$. The equation is given as follows:

$$
d_{M}\left(v_{0}\right)=\sum_{i} \mathbb{1}_{v_{0} \in M_{i}}\left(v_{0}\right)
$$

where $\mathbb{1}_{v_{0} \in M_{i}}\left(v_{0}\right)$ is an indicator function for each $v_{0} \in M_{i}$. If the motif is an edge, then the node motif $M$-degree is the 
traditionally defined node degree. We measure the importance of the node by counting the motifs instead of the edges. In Figure 3, $d_{M}\left(v_{2}\right)$ and $d_{M}\left(v_{3}\right)$ are 4 and 3, respectively, which correctly reflect the importance of the nodes.

Using a central node or an important node as a seed is a prudent solution. However, in community search, a community should be found for any given node. This solution might not designate a major node as a seed, and the resulting community might be inaccurate. To improve the results, we propose a new seed-processing strategy that can handle any node.

If only one node is used as a seed, then the node that joins at the beginning might be a sparse node. Currently, the most commonly used method is to select a node and its neighbor node as seeds [11], [21], [34]. However, this method might introduce nodes that do not contribute. In Figure 3, for example, the neighboring seed set of $v_{0}$ is $\left\{v_{0}, v_{1}, v_{2}, v_{3}, v_{7}\right\}$, where $v_{7}$ has no contribution. In local higher-order graph clustering, we introduce a new seed strategy known as the motif seed, which is defined as follows.

Definition 1. In graph $G$, given a vertex $v_{0}$ and motif $M$, motif seed is a motif set that contains node $v_{0}$. The equation is as follows:

$$
\text { Motif Seed }=\bigcup_{v_{0} \in M_{i}} M_{i} .
$$

As shown in Figure 3, the motif seed of node $v_{0}$ is $\left\{v_{0}, v_{1}, v_{2}, v_{3}\right\}$. In Algorithm 1, after obtaining the motif seed, we examine the set and remove several negatively affected nodes. Notably, the seed in this case is an initial structure that contains a cluster of nodes and edges and is no longer a collection of nodes. If we do not specify the original node, then we use the node with a motif $M$-degree that is higher than the original node, and add the motif $M$ that contains the original node to the seed. We experimentally prove that using this strategy to select seeds is effective.

\section{B. Expansion phase}

Once we obtain the original cluster, we intend to expand the cluster around this region. The traditional extension method selects a node from the cluster neighbor nodes to join the cluster, which can cause the fitness function to obtain the maximum and positive value until the neighbor node no longer increases or no neighbor node exists. However, certain different situations occur in higher-order networks, i.e., they use the motif as a unit. When only one node is added at each time, a network is likely to fall into a local pause or no suitable cluster might be found. Once $k-1$ nodes are added at each time, considerable time is required to obtain the optimal combination.

In this work, we propose a new method based on the characteristics of multiple nodes in a higher-order network. In the beginning, we add only one node to the cluster, i.e., we select a node from the neighbors of the cluster that causes the fitness function to take on a maximum and positive value to join the cluster. If all of the joined nodes are negative, then we consider adding two nodes at a time. We also consider making the fitness function take on the maximum and positive value for both nodes. Otherwise, we add more nodes until we obtain $k-1$ nodes. If the fitness function no longer increases or no neighbor node exists, then the expansion phase is stopped. To save computing costs, multiple connected nodes are added at one time.

We use the local motif rate $R_{M}$ as the fitness function. The equation is given as follows:

$$
R_{M}^{A_{i}}\left(C \cup A_{i}\right)=R_{M}\left(C \cup A_{i}\right)-R_{M}(C),
$$

where $C$ represents a cluster. To ensure that the calculation is not excessively large, $A_{i}$ denotes a set of respective adjacent nodes of size $i$ in which at least one node is a neighbor of cluster $C, i$ ranges from 1 to $k-1$, and $R_{M}\left(C \cup A_{i}\right)$ and $R_{M}(C)$ represent the local motif rate when the cluster node set is $C \cup A_{i}$ and $C$. This equation represents the gain obtained by adding node set $A_{i}$ to cluster $C$, and $A_{i}$ is added to the cluster to make the fitness function obtain a maximum and positive value.

To calculate $R_{M}\left(C \cup A_{i}\right)$, we do not need to recount the number of motifs each time and can directly use the $R_{M}(C)$ solution. Only the motif related to $A_{i}$ should be calculated. We use $E_{i n}\left(A_{i}\right)$ to represent the number of instances of motif $\mathrm{M}$ between $A_{i}$ and $C$,

$$
E_{i n}\left(A_{i}\right)=\operatorname{links}\left(C, A_{i}\right)
$$

$E_{\text {out }}\left(A_{i}\right)$ represents the number of instances of motif $\mathrm{M}$ between $A_{i}$ and $\bar{C}$,

$$
E_{\text {out }}\left(A_{i}\right)=\operatorname{links}\left(\bar{C}, A_{i}\right)
$$

In Figure $4 \mathrm{~A}, A_{i}$ is $\left\{v_{0}\right\}$; and $E_{\text {in }}\left(A_{i}\right)$ and $E_{\text {out }}\left(A_{i}\right)$ are the numbers of blue and green triangles, respectively, i.e., 1 and 2. In Figure $4 \mathrm{~B}, A_{i}$ is $\left\{v_{0}, v_{1}\right\}$; and $E_{\text {in }}\left(A_{i}\right)$ and $E_{\text {out }}\left(A_{i}\right)$ are 3 and 4 , respectively. From Eqs. (3), (5), and (6), we can obtain the following expression:

$$
R_{M}\left(C \cup A_{i}\right)=\frac{N_{\text {in }}(C)+E_{\text {in }}\left(A_{i}\right)}{\left(N_{\text {in }}(C)+N_{\text {out }}(C)+E_{\text {out }}\left(A_{i}\right)\right)^{\alpha}} .
$$

$N_{\text {in }}(C)$ and $N_{\text {out }}(C)$ have been derived in $R_{M}(C)$, and only $E_{\text {out }}\left(A_{i}\right)$ and $E_{\text {in }}\left(A_{i}\right)$ are calculated. We can simply prove that Eq. (7) is established.

Proof: For the number of intra-cluster motifs, according to Eqs. (1) and (5),

$$
\begin{aligned}
N_{i n}\left(C \cup A_{i}\right) & =\operatorname{links}_{M}\left(C \cup A_{i}, C \cup A_{i}\right) \\
& =\operatorname{links}_{M}(C, C)+\operatorname{links} M\left(C, A_{i}\right)+\operatorname{links} s_{M}\left(A_{i}, A_{i}\right) \\
& =N_{i n}(C)+E_{i n}\left(A_{i}\right)+N_{i n}\left(A_{i}\right) .
\end{aligned}
$$

Given that $\left|A_{i}\right|<|M|$, i.e., no motif $\mathrm{M}$ exists in $A_{i}, N_{i n}\left(A_{i}\right)=$ 0 . The final result is

$$
N_{i n}\left(C \cup A_{i}\right)=N_{i n}(C)+E_{i n}\left(A_{i}\right)
$$

For the number of inter-cluster motifs, according to Eqs. (2), (5), and (6),

$$
\begin{aligned}
N_{\text {out }}\left(C \cup A_{i}\right) & =\operatorname{links}_{M}\left(C \cup A_{i}, \overline{C \cup A_{i}}\right) \\
& =\operatorname{links}_{M}(C, \bar{C})-\operatorname{links} s_{M}\left(C, A_{i}\right)+\operatorname{links} s_{M}\left(\bar{C}, A_{i}\right) \\
& =N_{\text {out }}(C)-E_{\text {in }}\left(A_{i}\right)+E_{\text {out }}\left(A_{i}\right)
\end{aligned}
$$




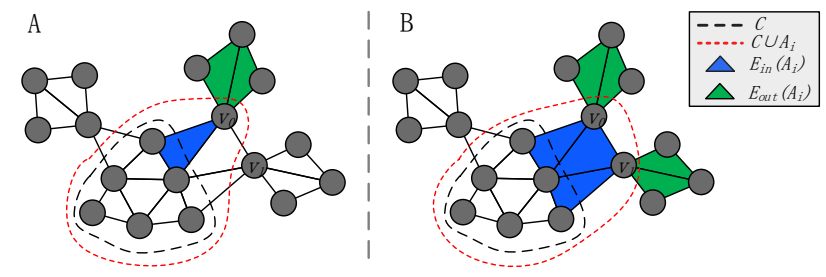

Fig. 4. Examples of calculating $R_{M}\left(C \cup A_{i}\right)$ when the motif is a triangle and $R_{M}(C)$ is $4 /(4+4)^{\alpha}$. A: Add $\left\{v_{0}\right\}, R_{M}\left(C \cup A_{1}\right)$ is $(4+1) /(4+4+2)^{\alpha}$. B: Add $\left\{v_{0}, v_{1}\right\}, R_{M}\left(C \cup A_{2}\right)$ is $(4+3) /(4+4+4)^{\alpha}$.

For the local motif rate, according to Eqs. (3), (8), and (9), we can obtain

$$
\begin{aligned}
R_{M}\left(C \cup A_{i}\right) & =\frac{N_{\text {in }}\left(C \cup A_{i}\right)}{\left(N_{\text {in }}\left(C \cup A_{i}\right)+N_{\text {out }}\left(C \cup A_{i}\right)\right)^{\alpha}} \\
& =\frac{N_{\text {in }}(C)+E_{\text {in }}\left(A_{i}\right)}{\left(N_{\text {in }}(C)+N_{\text {out }}(C)+E_{\text {out }}\left(A_{i}\right)\right)^{\alpha}} .
\end{aligned}
$$

Therefore we only need to recalculate $E_{\text {in }}\left(A_{i}\right)$ and $E_{\text {out }}\left(A_{i}\right)$ when obtaining $R_{M}\left(C \cup A_{i}\right)$.

In Figure 4, we provide two examples of calculating $R_{M}(C \cup$ $\left.A_{i}\right) . N_{\text {in }}(C)$ and $N_{\text {out }}(C)$ are equal to 4 , and $R_{M}(C)$ is $4 /(4+$ $4)^{\alpha}$. In Figure $4 \mathrm{~A}, A_{i}$ is $\left\{v_{0}\right\}$. From Eq. (5) and (6), we can only calculate $E_{\text {in }}\left(A_{i}\right)$ and $E_{\text {out }}\left(A_{i}\right)$, which are equal to 1 and 2, respectively, and $R_{M}\left(C \cup A_{i}\right)$ is $(4+1) /(4+4+2)^{\alpha} . R_{M}\left(C \cup A_{i}\right)$ is less than $R_{M}(C)$, and thus, $A_{i}$ cannot be expanded to $C$. In Figure $4 \mathrm{~B}, A_{i}$ is $\left\{v_{0}, v_{1}\right\} . E_{\text {in }}\left(A_{i}\right)$ and $E_{\text {out }}\left(A_{i}\right)$ are equal to 3 and 4 , respectively. We can obtain $R_{M}\left(C \cup A_{i}\right)$ as $(4+3) /(4+$ $4+4)^{\alpha}$, which is greater than $R_{M}(C)$.

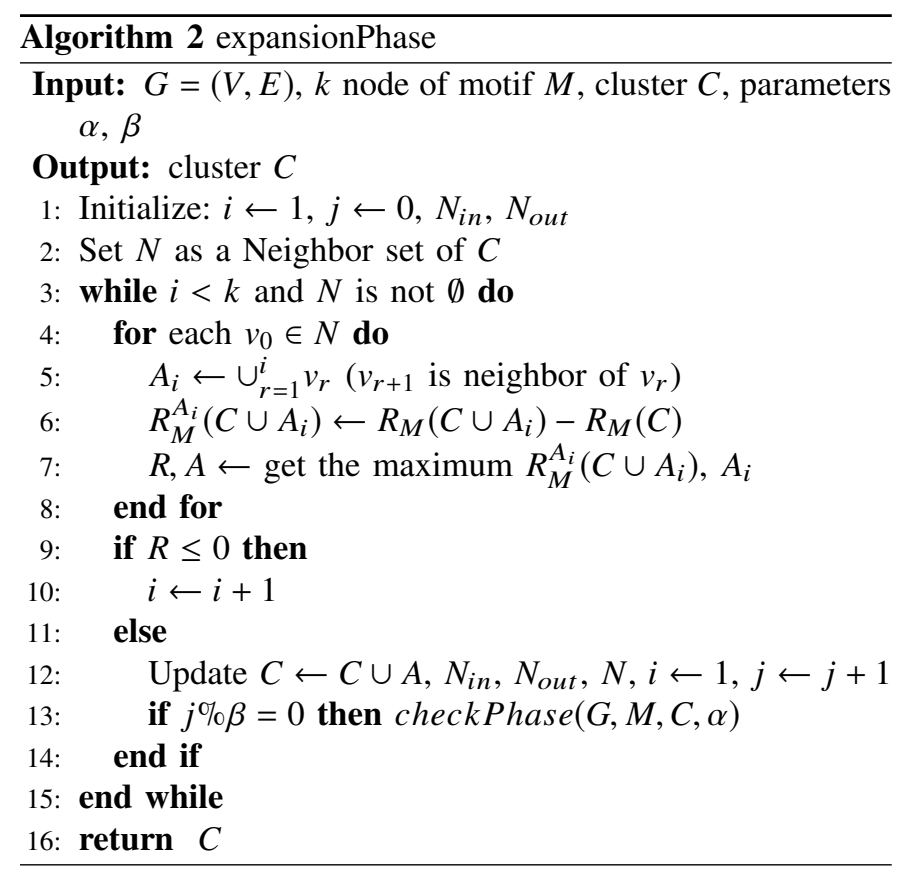

We use Algorithm 2 to describe the extension phase, and the concrete steps are explained as follows.

1) In lines 1 and 2 , the variables $i$ and $j$ are initialized to 1 and 0 , respectively, where $\mathrm{i}$ represents the scale of $A_{i}$ and $\mathrm{j}$ represents the number of extensions. $N_{i n}$ and $N_{\text {out }}$ are calculated, and $N$ is established to be a set of neighbor nodes of $C$.
2) In lines 4-8, the maximum value of $R_{M}^{A_{i}}\left(C \cup A_{i}\right)$ and its corresponding $A_{i}$ are obtained as $R$ and $A$, respectively. To reduce the combination, $A_{i}$ is a set of neighboring nodes and $\left|A_{i}\right|$ is $i$.

3) In lines $9-14$, when $R$ is less than 0 , the value of $i$ is increased, i.e., the number of extended nodes is increased. Otherwise, the cluster $C \cup A$ is set to $C$, and the values of $N_{i n}, N_{\text {out }}, N, i$, and $j$ are updated. In line 13 , cluster $C$ is checked every $\beta$ times, which is an option, and a larger $\beta$ value can be set to obtain a shorter time.

\section{Check phase}

When the nodes in a cluster continue to increase, previous nodes might have a negative effect cluster quality. To address the negative impact, the check phase was added. This phase is similar to the expansion phase, and both aim to increase cluster quality, but the difference is that nodes are removed in the latter.

To ensure that the calculation is not too large, our algorithm only checks a single node, i.e., whether the local motif rate increases after temporarily removing a node in the cluster. If the rate increases, then we delete it, and otherwise, we preserve it. For specific methods, please refer to Algorithm 3 . The equation is given as follows:

$$
R_{M}^{v_{0}}\left(C-\left\{v_{0}\right\}\right)=R_{M}\left(C-\left\{v_{0}\right\}\right)-R_{M}(C)
$$

Similar to Eq. (7), Eq. (10) can also take advantage of the results of previous calculations, as long as the motif that contains node $v_{0}$ is calculated. The calculation method is

$$
R_{M}\left(C-\left\{v_{0}\right\}\right)=\frac{N_{\text {in }}(C)-E_{\text {in }}\left(\left\{v_{0}\right\}\right)}{\left(N_{\text {in }}(C)+N_{\text {out }}(C)-E_{\text {out }}\left(\left\{v_{0}\right\}\right)\right)^{\alpha}},
$$

and the proof is as follows.

Proof: For the number of intra-cluster motifs,

$$
\begin{aligned}
N_{\text {in }}\left(C-\left\{v_{0}\right\}\right) & =\operatorname{links}_{M}\left(C-\left\{v_{0}\right\}, C-\left\{v_{0}\right\}\right) \\
& =\operatorname{links}_{M}(C, C)-\operatorname{links}_{M}\left(C, v_{0}\right) \\
& =N_{\text {in }}(C)-E_{\text {in }}\left(\left\{v_{0}\right\}\right) .
\end{aligned}
$$

For the number of inter-cluster motifs,

$$
\begin{aligned}
N_{\text {out }}\left(C-\left\{v_{0}\right\}\right) & =\operatorname{links}_{M}\left(C-\left\{v_{0}\right\}, \overline{C-\left\{v_{0}\right\}}\right) \\
& =\operatorname{links}_{M}(C, \bar{C})+\operatorname{links}_{M}\left(C,\left\{v_{0}\right\}\right)-\operatorname{link} s_{M}\left(\bar{C},\left\{v_{0}\right\}\right) \\
& =N_{\text {out }}(C)+E_{\text {in }}\left(\left\{v_{0}\right\}\right)-E_{\text {out }}\left(\left\{v_{0}\right\}\right)
\end{aligned}
$$

For the local motif rate, according to Eqs. (3), (12), and (13),

$$
\begin{aligned}
R_{M}\left(C-\left\{v_{0}\right\}\right) & =\frac{N_{\text {in }}\left(C-\left\{v_{0}\right\}\right)}{\left(N_{\text {in }}\left(C-\left\{v_{0}\right\}\right)+N_{\text {out }}\left(C-\left\{v_{0}\right\}\right)\right)^{\alpha}} \\
& =\frac{N_{\text {in }}(C)-E_{\text {in }}\left(\left\{v_{0}\right\}\right)}{\left(N_{\text {in }}(C)+N_{\text {out }}(C)-E_{\text {out }}\left(\left\{v_{0}\right\}\right)\right)^{\alpha}} .
\end{aligned}
$$

Therefore, Eq. (11) is established. 


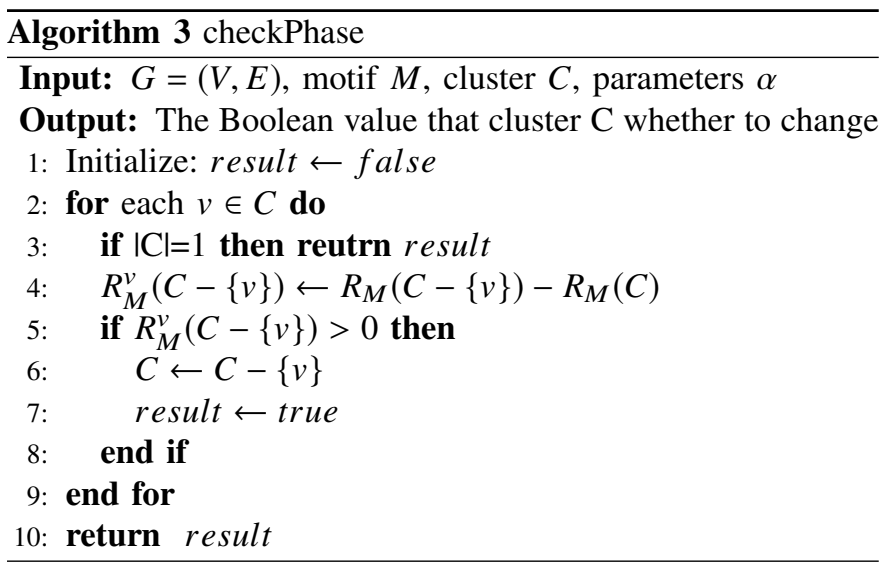

\section{Time complexity analysis}

We analyze the time complexity of the MLEO algorithm. Given a graph, $n$ nodes, $m$ edges, and the degree of each node is $d=\left\{d_{1}, \cdots, d_{n}\right\}$, where the maximum degree is $d_{\max }$. For Algorithm 2, when maximizing the fitness of neighbors, the time complexity of sorting them is $d_{1} \log d_{1}$. Subsequent extensions only consider the insert sequence. In the worst case, the remaining $n-1$ nodes are added to the cluster, which is $\sum_{i=2}^{n} \log d_{i}$. For the motifs of k nodes, the result must be multiplied by $k-1$. Using simple scaling calculations,

$$
\begin{aligned}
& (k-1) d_{1} \log d_{1}+(k-1) \sum_{i=2}^{n} \log d_{i} \\
& \leq k d_{\text {max }} \log d_{\text {max }}+k(n-1) \log d_{\text {max }} \\
& \leq k(2 n-1) \log d_{\text {max }} .
\end{aligned}
$$

The complexity of Algorithm 2 is $O\left(k n \delta \log d_{\max }\right)$, where $\delta$ is the complexity of finding a motif. Furthermore, the complexity of Algorithm 1 is $O\left(k n^{2} \delta \log d_{\max }\right)$. The complexity of MAPPR is $O\left(\delta_{\text {all }}+m \log ^{4} m / \phi^{2}\right)$, where $\phi$ is conductance, and $\delta_{\text {all }}$ represents the cost of constructing $\mathrm{W}$, which must identify the motif of the entire graph, but our method only needs to identify the relevant region. The problem of identifying motifs and counting them is a highly challenging task, and it is NP-complete [35]. Many studies focus on quickly identifying motifs in the network [36]-[38].

\section{EXPERIMENTS}

In Section IV-A, we discuss the evaluation metrics. In Section IV-B, we introduce the dataset in the experiment. In Section IV-C, we evaluate the MLEO algorithm in directed and undirected networks. In a directed network, we set $\alpha=1$ and use a variety of motifs to determine the degree of clustering to the local motif rate. In an undirected network, we use $F_{1}$, recall, and precision as measures.

In Section IV-D, we use each node in the network as the original seed and compare different seed strategies. Simultaneously, we count the clustering results that correspond to different node motif $M$-degrees. In Section IV-E, we use different $\alpha$ values to find clusters of different sizes, which proves that the proposed algorithm is also feasible for hierarchical clustering.

\section{A. Evaluation metrics}

In using our proposed evaluation metrics local motif rate to evaluate the degree of clustering, we also adopt the universal evaluation metric $F_{1}$ to evaluate ground truth clusters. Formally, given $C$ and $C^{\prime}$ as two arbitrary clusters in the same network, the $F_{1}$ score of $C$ in matching with $C^{\prime}$ is defined as follows;

$$
F_{1}=\frac{2 \times \operatorname{precision}\left(C, C^{\prime}\right) \times \operatorname{recall}\left(C, C^{\prime}\right)}{\operatorname{precision}\left(C, C^{\prime}\right)+\operatorname{recall}\left(C, C^{\prime}\right)},
$$

where precision $\left(C, C^{\prime}\right)=\frac{\left|C \cap C^{\prime}\right|}{|C|}$ and $\operatorname{recall}\left(C, C^{\prime}\right)=\frac{\left|C \cap C^{\prime}\right|}{\left|C^{\prime}\right|}$.

$F_{1}$ is frequently used in supervised learning; however, the matching of clusters in unsupervised learning is more complicated. When we focus on the evaluation of a cluster, i.e., the performance evaluation of a cluster $C_{i}$ with the ground truth cluster set $C^{\prime}=\left\{C_{1}^{\prime}, \cdots, C_{Q}^{\prime}\right\}$, we divide the $F_{1}$ score into a maximum matching $F_{1-\max }$ and an average matching $F_{1-a v e .}$

$$
\begin{aligned}
F_{1-\text { max }}\left(C_{i}, C^{\prime}\right) & =\max _{j: 1 \rightarrow Q} F_{1}\left(C_{i}, C_{j}^{\prime}\right), \\
F_{1-\text { ave }}\left(C_{i}, C^{\prime}\right) & =\text { average }_{j: 1 \rightarrow Q, C_{i} \cup C_{j}^{\prime} \neq \emptyset} F_{1}\left(C_{i}, C_{j}^{\prime}\right) .
\end{aligned}
$$

$F_{1-\max }$ and $F_{1-\text { ave }}$ are both in $[0,1]$; the closer to 1 , the better the result. $F_{1-\max }$ calculates the $F_{1}$ score that matches the cluster best in the result set, whereas $F_{1-a v e}$ calculates the average $F_{1}$ of all the sets that intersect with the ground truth clusters. In the experiment, we obtain the average of the $F_{1}$ that correspond to clusters $C=\left\{C_{1}, \cdots, C_{P}\right\}$, including $F_{1-\max }$ and $F_{1-a v e}$, which are calculated as follows:

$$
\begin{aligned}
& F_{1-\max }\left(C, C^{\prime}\right)=\frac{1}{P} \sum_{i=1}^{P} F_{1-\max }\left(C_{i}, C^{\prime}\right), \\
& F_{1-\text { ave }}\left(C, C^{\prime}\right)=\frac{1}{P} \sum_{i=1}^{P} F_{1-\text { ave }}\left(C_{i}, C^{\prime}\right) .
\end{aligned}
$$

\section{B. Dataset}

Several different real network datasets from the Stanford Network Analysis Project [40] are used in the experimental comparison, including 4 ground-truth community undirected networks, i.e., com-dblp, com-amazon, com-youtube and com-lj, and 6 directed networks, i.e., Florida-bay, Celegans-frontal, Email-Eu-core, Cit -hepPh, Soc-Slashdot0811 and Web-Stanford. The details of each network are shown in Table I.

The LFR [41] is a model for generating benchmark datasets to synthesize the network. The LFR benchmark network has been widely adopted and supplies researchers with the flexibility to control network topologies by adjusting different parameters. An important parameter in this model is the mixing parameter $\mu$. In the LFR model, each node shares a fraction $1-\mu$ of its links with the other nodes of its community and a fraction $\mu$ with the other nodes of the network. Therefore, if the value of $\mu$ is closer to 1 in the benchmark network, then it is more difficult to distinguish the community. We synthesize different networks by changing the mixing parameters and fixing other parameters. The fixed parameters including the number of nodes $n$ is 10000 , the 
TABLE I

AN INTRODUCTION TO THE REAL NETWORK IN THE EXPERIMENT [39].

\begin{tabular}{lllll}
\hline Name & Type & Nodes & Edges & Description \\
\hline Com-dblp & Undirected, Communities & 317080 & 1049866 & DBLP collaboration network \\
Com-amazon & Undirected, Communities & 334863 & 925872 & Amazon product network \\
Com-youtube & Undirected, Communities & 1134890 & 2987624 & Youtube online social network \\
Com-lj & Undirected, Communities & 3997962 & 34681198 & LiveJournal online social network \\
Florida-bay & Directed & 128 & 2106 & Florida Bay food web \\
C-elegans-frontal & Directed & 131 & 764 & Frontal neuronal connections in C. elegans \\
Email-Eu-core & Directed & 1005 & 25571 & E-mail network \\
Cit-HepPh & Directed & 34546 & 421578 & Arxiv High Energy Physics paper citation network \\
Soc-Slashdot0811 & Directed & 77360 & 905463 & Slashdot social network from November 2008 \\
Web-Stanford & Directed & 281903 & 2312497 & Web graph of Stanford.edu \\
\hline
\end{tabular}

TABLE II

COMPARISON BETWEEN THE MLEO ALGORITHM AND THE MAPPR ALGORITHM, WHERE THE MOTIF IS A TRIANGLE. BOTH ALGORITHMS USE RANDOMLY SELECTED SEEDS. A TOTAL OF 100 COMMUNITIES WITH MORE THAN 50 NODES ARE CONSIDERED AND $F_{1-a v e}$ IS COMPARED.

\begin{tabular}{|c|c|c|c|c|c|c|c|c|c|}
\hline \multirow{2}{*}{ Networks } & \multirow{2}{*}{$|\mathrm{V}|$} & \multirow{2}{*}{$|\mathrm{E}|$} & \multirow{2}{*}{$\begin{array}{c}\mid \mathrm{Cl} \\
(\text { size }>50)\end{array}$} & \multicolumn{2}{|c|}{$F_{1-a v e}$} & \multicolumn{2}{|c|}{ precision ave $_{\text {a }}$} & \multicolumn{2}{|c|}{ recall ave } \\
\hline & & & & MAPPR & MLEO & MAPPR & MLEO & MAPPR & MLEO \\
\hline com-dblp & $317 \mathrm{~K}$ & $1.05 \mathrm{M}$ & 100 & 0.167 & 0.224 & 0.197 & 0.666 & 0.225 & 0.155 \\
\hline com-amazon & $335 \mathrm{~K}$ & $926 \mathrm{~K}$ & 100 & 0.648 & 0.684 & 0.838 & 0.924 & 0.555 & 0.587 \\
\hline com-youtube & $1.13 \mathrm{M}$ & $2.99 \mathrm{M}$ & 100 & 0.232 & 0.331 & 0.270 & 0.371 & 0.303 & 0.430 \\
\hline com-lj & $4.00 \mathrm{M}$ & $34.7 \mathrm{M}$ & 100 & 0.251 & 0.330 & 0.182 & 0.266 & 0.556 & 0.536 \\
\hline
\end{tabular}

TABLE III

COMPARISON BETWEEN THE MLEO ALGORITHM AND THE MAPPR ALGORITHM, WHERE THE MOTIF IS A TRIANGLE. BOTH ALGORITHMS USE RANDOMLY SELECTED SEEDS. A TOTAL OF 100 COMMUNITIES WITH MORE THAN 50 NODES ARE CONSIDERED AND $F_{1-\text { max }}$ IS COMPARED.

\begin{tabular}{cccccccccc}
\hline \multirow{2}{*}{ Networks } & \multirow{2}{*}{$|\mathrm{V}|$} & \multirow{2}{*}{$\mathrm{IE|}$} & \multirow{2}{*}{$\mathrm{|C|}$} & \multicolumn{2}{c}{$F_{1-\max }$} & \multicolumn{2}{c}{ precision $_{\max }$} & \multicolumn{2}{c}{ recall $_{\max }$} \\
& & & $($ size $>50)$ & MAPPR & MLEO & MAPPR & MLEO & MAPPR & MLEO \\
\hline com-dblp & $317 \mathrm{~K}$ & $1.05 \mathrm{M}$ & 100 & 0.406 & $\mathbf{0 . 6 0 3}$ & 0.314 & $\mathbf{0 . 7 4 8}$ & $\mathbf{0 . 8 5 3}$ & 0.618 \\
com-amazon & $335 \mathrm{~K}$ & $926 \mathrm{~K}$ & 100 & 0.940 & $\mathbf{0 . 9 6 0}$ & 0.914 & $\mathbf{0 . 9 5 1}$ & $\mathbf{0 . 9 8 2}$ & 0.976 \\
com-youtube & $1.13 \mathrm{M}$ & $2.99 \mathrm{M}$ & 100 & 0.440 & $\mathbf{0 . 5 0 0}$ & 0.390 & $\mathbf{0 . 4 6 5}$ & 0.711 & $\mathbf{0 . 7 4 4}$ \\
com-lj & $4.00 \mathrm{M}$ & $34.7 \mathrm{M}$ & 100 & 0.459 & $\mathbf{0 . 5 2 9}$ & 0.318 & $\mathbf{0 . 3 9 1}$ & $\mathbf{0 . 9 9 1}$ & 0.964 \\
\hline
\end{tabular}

average degree is 30 , the maximum degree is 50 , and the community size ranges from 50 to 500 .

\section{Recovering communities in networks using MLEO}

We compare the MLEO and MAPPR algorithms on real networks with the ground truth communities. We analyze the ground truth community on several well-known networks, including com-dblp, com-amazon, com-youtube, and comLiveJournal. For each network, we use a triangle (shown in Figure 1) as a motif and randomly select nodes as seeds to examine 100 communities with more than 50 nodes. The experimental data sets and results are presented in Tables II and III. The experimental results demonstrate that our algorithm performs better in terms of $F_{1}$ and precision with the ground truth community in these networks than MAPPR, and its recall is also competitive.

We also examine different motifs in several directed real networks and randomly select 50 seeds from the network to obtain the average local motif rate (without special explanation, the following experiment generally sets $\alpha$ to 1) of the community. The datasets include Florida-bay, C-elegansfrontal, email-Eu-core, cit-HepPh, soc-Slashdot0811, and webStanford. The results are shown in Table IV. There is no
$M_{4}$ in the Florida-bay network, and thus, it is indicated by a horizontal line.

The references [23], [26] offer some selected interesting experiments on Florida Bay. To further demonstrate our algorithm, we search for higher-order graph clustering of the Florida-bay food web, which represents the carbon exchange in Florida Bay. In this work, we use $M_{7}$ as the motif, i.e., we examine the clustering of this carbon exchange pattern in this network. As shown in Figure 5A, a total of 57 nodes, including $M_{7}$, are grouped into 5 clusters. To illustrate the result clearly, we use Figure 5B to represent the purple clusters in Figure 5A. This higher-order cluster shows that Eels and Lizardfish eat each other, and they are food for several animals. Nodes 100 (Loon), 103 (Comorant), 110 (Predatory Ducks), 111 (Raptors) are overlapping nodes of the purple and green communities. These animals can both prey on fish and have more food sources on the shore.

\section{Obtaining good seeds}

We use several motifs to compare different seed strategies under different $\mu$ LFR and real networks, including original seeds, neighbor seeds, and motif seeds. The results are presented in Figure 6 and Table V. Overall, the motif seed is better. However, when we use a triangle as a motif in 
TABLE IV

RANDOMLY SELECTED 50 NODES FROM THE REAL NETWORK AS SEEDS TO OBTAIN THE AVERAGE LOCAL MOTIF RATE $(\alpha=1)$ OF THE COMMUNITY, WHERE THE MOTIF IS $M_{1}-M_{7}$.

\begin{tabular}{cccccccccc}
\hline \multirow{2}{*}{ Networks } & \multirow{2}{*}{$|\mathrm{V}|$} & \multirow{2}{*}{$|\mathrm{E}|$} & $\boldsymbol{M}_{1}$ & $\boldsymbol{M}_{2}$ & $\boldsymbol{M}_{3}$ & $\boldsymbol{M}_{4}$ & $\boldsymbol{M}_{5}$ & $\boldsymbol{M}_{6}$ & $\boldsymbol{M}_{7}$ \\
\hline Florida-bay & 128 & 2106 & 0.943 & 0.766 & 1.000 & - & 0.662 & 0.706 & 0.692 \\
C-elegans-frontal & 131 & 764 & 0.928 & 0.781 & 0.733 & 0.850 & 0.415 & 0.523 & 0.652 \\
email-Eu-core & 1005 & 25571 & 0.492 & 0.322 & 0.404 & 0.445 & 0.346 & 0.300 & 0.362 \\
cit-HepPh & 34546 & 421578 & 0.943 & 0.935 & 0.992 & 1.000 & 0.392 & 0.758 & 0.774 \\
soc-Slashdot0811 & 77360 & 905468 & 0.695 & 0.329 & 0.227 & 0.326 & 0.240 & 0.265 & 0.292 \\
web-Stanford & 281903 & 23124907 & 0.889 & 0.703 & 0.896 & 0.884 & 0.441 & 0.829 & 0.560 \\
\hline
\end{tabular}

TABLE V

SEED STRATEGY ON REAL NETWORKS. OS, NS, AND MS REPRESENT ORIGINAL SEED, NEIGHBOR SEED, AND MOTIF SEED.

\begin{tabular}{|c|c|c|c|c|c|c|c|c|c|c|c|c|}
\hline \multirow{3}{*}{ Network } & \multicolumn{6}{|c|}{$F_{1-a v e}$} & \multicolumn{6}{|c|}{$F_{1-\max }$} \\
\hline & \multicolumn{3}{|c|}{ Triangle } & \multicolumn{3}{|c|}{ Quadrangle } & \multicolumn{3}{|c|}{ Triangle } & \multicolumn{3}{|c|}{ Quadrangle } \\
\hline & OS & NS & MS & OS & NS & MS & OS & NS & MS & OS & NS & MS \\
\hline com-dblp & 0.224 & 0.181 & 0.182 & 0.234 & 0.271 & 0.297 & 0.603 & 0.549 & 0.562 & 0.453 & 0.509 & 0.538 \\
\hline com-amazon & 0.684 & 0.703 & 0.705 & 0.490 & 0.574 & 0.582 & 0.960 & 0.960 & 0.966 & 0.907 & 0.907 & 0.909 \\
\hline
\end{tabular}

A

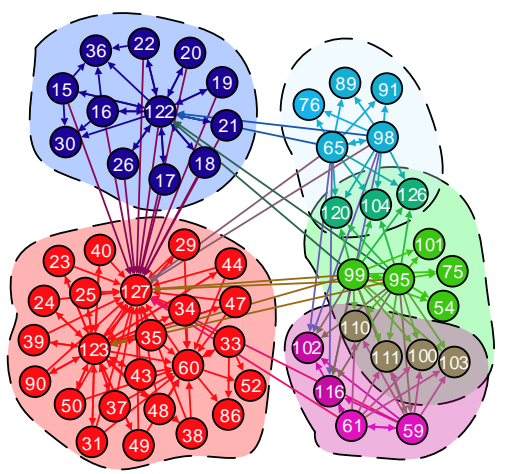

B

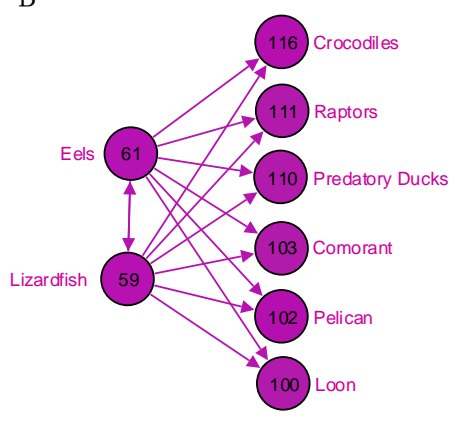

Fig. 5. $M_{7}$ is used for higher-order graph clustering of the Florida-bay food web. A: 57 nodes containing motif $M_{7}$. The algorithm finds 5 clusters and uses different colors for representation. B: Clear illustration of the purple community in A.

com-dblp, the original seed exhibits better community effect. We hypothesize that this result is related to seed size and community size. The size of the community that the neighbor seed or motif seed has obtained is two to three times that of the original seed. In synthetic networks, when triangles are used as motifs, motif seed and neighbor seed exhibit similar effects and are superior to the original seed. Considering the special nature of triangles, the resulting motif seed is a subset of the neighbor seed. We use a quadrangle (as shown in Figure 1) as the motif. The results indicate that the motif seed is similar to the neighbor seed when $\mu$ is less than or equal to 0.45 . However, when $\mu$ is greater than 0.45 , the effect is considerably better than that of the neighbor seed. We speculate that the motif seed is more effective than the neighbor seed in the case of more complex incomplete graphs because finding complex motifs is a complicated problem itself. We plan to complete this experiment in the future.

We use triangle and quadrangle as motifs, and adopt the original seed strategy for all nodes in several LFR models; that is, we do not process the seeds and use the MLEO algorithm directly for each node. We calculate the distribution of nodes with different $M$-degrees and the average local motif rate and
$F_{1}$. The result is shown in Figure 7. Using a triangle as the motif, as shown in Figures 7A and 7B, the trends of the local motif rate and $F_{1}$ are highly similar and even notably close at many values. In Figures 7C and 7D, quadrilaterals are used as motifs, and although the local motif rate and $F_{1}$ values are not close to each other, their trends and shapes are highly similar. In other words, our local motif rate can reflect the clustering results to a certain extent.

In Figure 7 , the overall trend of $F_{1}$ increases with an increase in $M$-degree and decreases locally. In Figure 7A, when the $M$-degree is 2 , the value of $F_{1}$ is notably high, which is equal to 1 , but there is only one such point. We use the other nodes in this cluster as seeds, and the experimental results show that most of the clusters have an $F_{1}$ value equal to 1 .

\section{E. Adjusting $\alpha$ to discover communities with different sizes}

To demonstrate that our algorithm can be applied to hierarchical clustering, we test it on a synthetic network with a built-in hierarchical community structure. We use a benchmark similar to the Andrea Lancichinetti [27] benchmark, which is a simple extension of the classic benchmark proposed by 

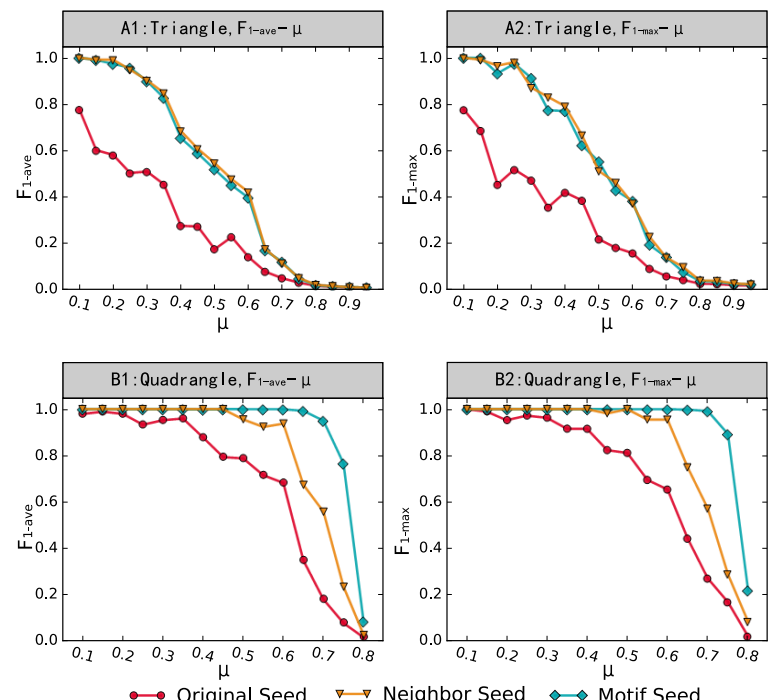

Fig. 6. Results of using different seed strategies in the LFR model. For each data set, 100 original seeds are randomly selected using different strategies. A: When a triangle is used as the motif, the motif seed and neighbor seed exhibit similar effects and are better than the original seed. B: The motif seed and neighbor seed are better than the original Seed, where the motif seed is the same as the neighbor seed when $\mu$ is less than or equal to 0.45 , but when the value of $\mu$ is larger, the motif seed effect is considerably better than that of the neighbor seed.

Girvan and Newman [42]. There are a total of 512 nodes, including 4 groups of benchmark data proposed by Girvan and Newman, with each group containing 128 nodes, which are divided into 4 communities, i.e., 32 nodes per community. We simply expand the data such that the edge density between the two sets of data is greater than that of the other two sets, as shown in Figure 8.

In the preceding network, a seed is selected and a triangle is used as motif. We change parameter $\alpha$, use the MLEO algorithm for clustering and subsequently observe the change in cluster size with parameter $\alpha$. The result is shown in Figure 9. We also apply the algorithm to synthetic and real networks. The result is shown in Figure 10. As $\alpha$ increases, the average cluster size decreases. Our algorithm can find clusters with different sizes after adjusting parameter $\alpha$. In Figure 9A, we increase the value of $\alpha$ with an increment of 0.01 . With the increases in parameter $\alpha$, the size of the cluster gradually decreases. To observe the changes further, we reduce the spacing and set it to 0.001, as shown in Figure 9B. After the spacing is decreased, we determine that the range of the $\alpha$ values corresponds to different sizes of clusters. When $\alpha$ is within the range of 0.15 to 1.52 , the cluster size is 32 , and its range is relatively large, thereby indicating that the community structure is relatively stable, which is consistent with the network proposed by Girvan and Newman. Proving the stability of a community structure still remains an open question, and we plan to investigate it in a future study.

\section{DISCUSSION AND CONCLUSION}

In this study, we propose the local motif rate to improve the density of motifs in a cluster for higher-order graph clustering. Compared with motif conductance, this rate is superior in
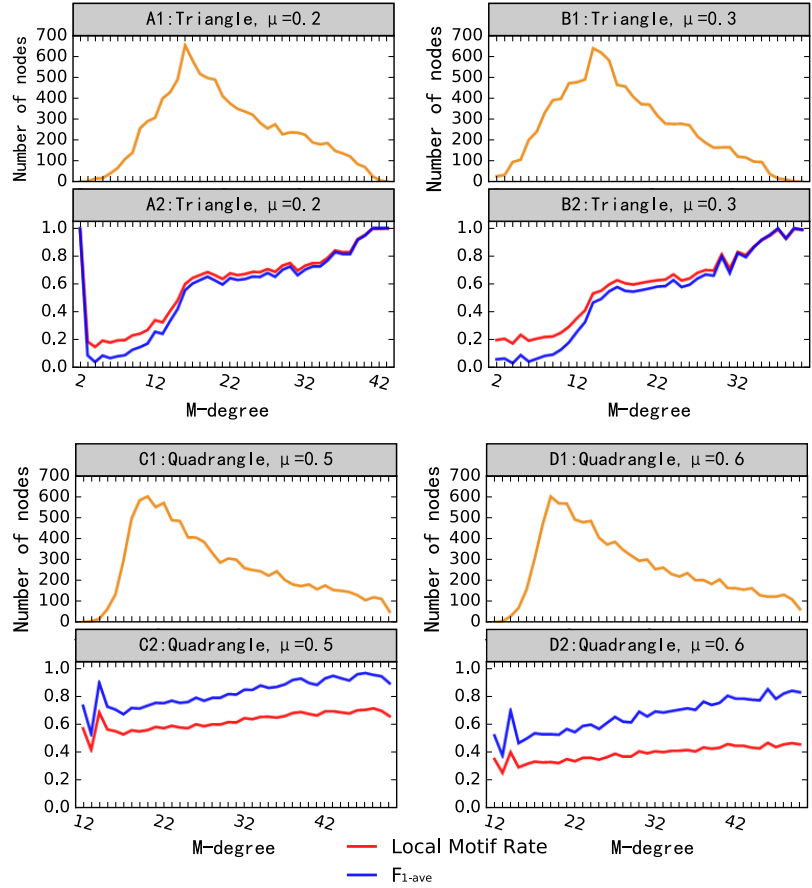

Fig. 7. Distribution and clustering results at different M-degrees. Triangles and quadrilaterals are used as motifs for clustering under several different LFR networks. In the four graphs, 1 denotes the distribution of M-degrees, and 2 denotes the average of the clustering results that correspond to all nodes as seeds in the M-degree.

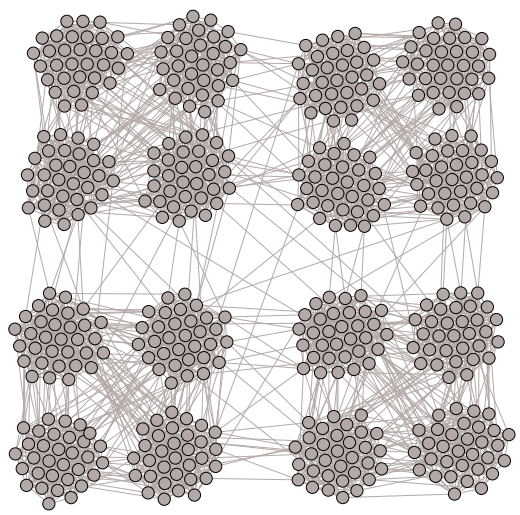

Fig. 8. Expansion of the 4 Girvan and Newman benchmark networks into a hierarchical network with 512 nodes.

measuring the degree of clustering in a local area. The experimental results show that our proposed method exhibits similar trends and shapes in terms of $F_{1}$ values and can respond to the degree of higher-order graph clustering. The local motif rate allows us to adjust the parameter $\alpha$ and control the size of a cluster. This approach can be applied to hierarchical clustering. Additionally, clustering stability can be estimated by adjusting the range of parameter $\alpha$ that corresponds to cluster size.

Furthermore, we propose a new seed strategy known as the motif seed. This type of seed strategy selects neighboring nodes directly as seeds when the complete graph is used as a motif, and our seed strategy works better when the incomplete subgraph is used as a motif. The current notion states that a 

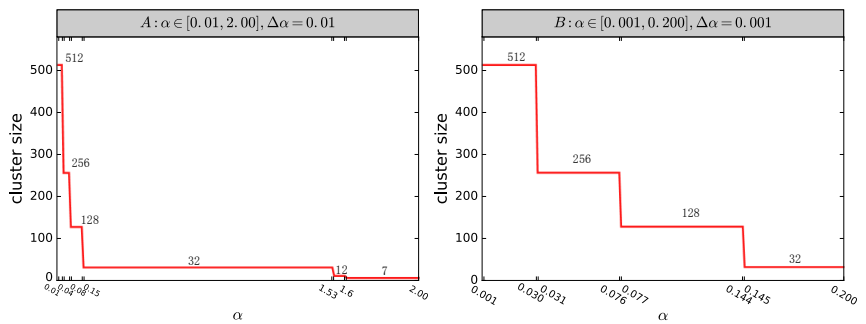

Fig. 9. Cluster size changes with parameter $\alpha$ under the MLEO algorithm when a triangle is used as the motif. A: The value of parameter $\alpha$ ranges from 0.01 to 2 , and the distance is 0.01 . B: The value of parameter $\alpha$ ranges from 0.001 to 0.2 , and the pitch is 0.001 .
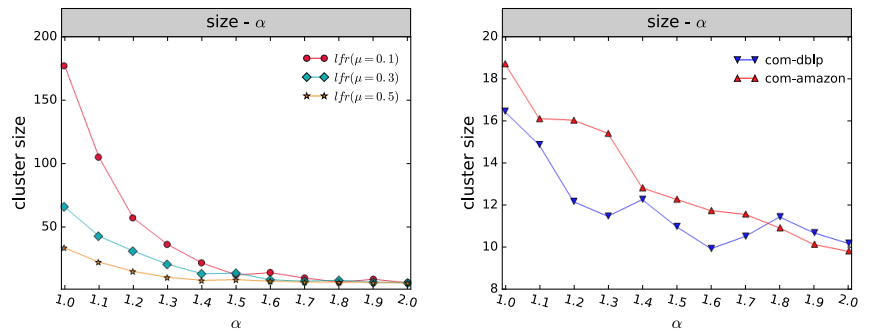

Fig. 10. Cluster size changes with parameter $\alpha$ in synthetic and real networks when a triangle is used as motif.

node with a higher degree can be used as a seed to obtain a better community, and thus, we compare the experimental findings under different M-degrees, showing that selecting nodes with a higher M-degree as seeds is more likely to achieve robust clusters.

In addition, we apply the concept of local extension optimization to higher-order graph clustering and propose an MLEO algorithm. This algorithm is a purely local method, and thus, it has no need to recognize the information of the entire network and can avoid the distortion caused by conversion to a weighted graph. In the experiment of directed and undirected graphs, we prove that the performance of MLEO can meet the standard of higher-order clustering. Higher-order graph clustering has a wide range of application scenarios. We can further compare the clusters with different motifs in the network to determine which collaboration model can achieve more extensive cooperation in scientific research.

\section{REFERENCES}

[1] T. He, L. Cai, T. Meng, L. Chen, Z. Deng, and Z. Cao, "Parallel Community Detection Based on Distance Dynamics for Large-Scale Network," IEEE Access, vol. 6, pp. 42 775-42 789, 2018.

[2] S. Fortunato and D. Hric, "Community detection in networks: A user guide," Physics Reports, vol. 659, pp. 1-44, Nov. 2016.

[3] T. Wang, J. Tan, W. Ding, Y. Zhang, F. Yang, J. Song, and Z. Han, "Inter-Community Detection Scheme for Social Internet of Things: A Compressive Sensing Over Graphs Approach," IEEE Internet of Things Journal, pp. 1-1, 2018.

[4] J.-S. Lee and T.-Y. Kao, "An Improved Three-Layer Low-Energy Adaptive Clustering Hierarchy for Wireless Sensor Networks," IEEE Internet of Things Journal, vol. 3, no. 6, pp. 951-958, Dec. 2016.

[5] M. Ren, J. Zhang, L. Khoukhi, H. Labiod, and V. Vèque, "A Unified Framework of Clustering Approach in Vehicular Ad Hoc Networks," IEEE Transactions on Intelligent Transportation Systems, vol. 19, no. 5, pp. 1401-1414, May 2018.
[6] R. S. Bali and N. Kumar, "Secure clustering for efficient data dissemination in vehicular cyber-physical systems," Future Generation Computer Systems, vol. 56, pp. 476-492, Mar. 2016.

[7] C. Chen, B. Wang, and R. Zhang, "Interference Hypergraph-Based Resource Allocation (IHG-RA) for NOMA-Integrated V2x Networks," IEEE Internet of Things Journal, vol. 6, no. 1, pp. 161-170, Feb. 2019.

[8] G. Xie, G. Zeng, J. Jiang, C. Fan, R. Li, and K. Li, "Energy management for multiple real-time workflows on cyber-physical cloud systems," Future Generation Computer Systems, May 2017.

[9] G. Xie, G. Zeng, Y. Liu, J. Zhou, R. Li, and K. Li, "Fast Functional Safety Verification for Distributed Automotive Applications During Early Design Phase," IEEE Transactions on Industrial Electronics, vol. 65, no. 5, pp. 4378-4391, May 2018.

[10] G. Xie, G. Zeng, R. Kurachi, H. Takada, R. Li, and K. Li, "Exact WCRT analysis for message-processing tasks on gateway-integrated in-vehicle CAN clusters," vol. 17, no. 6, pp. 95:1-95:29.

[11] J. J. Whang, D. F. Gleich, and I. S. Dhillon, "Overlapping Community Detection Using Neighborhood-Inflated Seed Expansion," IEEE Transactions on Knowledge and Data Engineering, vol. 28, no. 5, pp. 1272 1284, May 2016

[12] H. Yin, A. R. Benson, J. Leskovec, and D. F. Gleich, "Local higherorder graph clustering," in Proceedings of the 23rd ACM SIGKDD International Conference on Knowledge Discovery and Data Mining. ACM, 2017, pp. 555-564.

[13] I. M. Kloumann and J. M. Kleinberg, "Community membership identification from small seed sets," in Proceedings of the 20th ACM SIGKDD international conference on Knowledge discovery and data mining. ACM, 2014, pp. 1366-1375.

[14] S. Fortunato, "Community detection in graphs," Physics reports, vol. 486, no. 3-5, pp. 75-174, 2010.

[15] S. E. Schaeffer, "Graph clustering," Computer Science Review, vol. 1, no. 1, pp. 27-64, Aug. 2007.

[16] F. Havemann, M. Heinz, A. Struck, and J. Gläser, "Identification of overlapping communities and their hierarchy by locally calculating community-changing resolution levels," Journal of Statistical Mechanics: Theory and Experiment, vol. 2011, no. 01, p. P01023, 2011.

[17] L. Cai, T. Meng, T. He, L. Chen, and Z. Deng, "K-Hop community search based on local distance dynamics," in Lecture Notes in Computer Science (including subseries Lecture Notes in Artificial Intelligence and Lecture Notes in Bioinformatics), vol. 10638 LNCS, 2017, pp. 24 - 34.

[18] P. Li, H. Dau, G. Puleo, and O. Milenkovic, "Motif clustering and overlapping clustering for social network analysis," in INFOCOM 2017 IEEE Conference on Computer Communications. IEEE, 2017, pp. 1-9.

[19] R. Milo, S. Shen-Orr, S. Itzkovitz, N. Kashtan, D. Chklovskii, and U. Alon, "Network Motifs: Simple Building Blocks of Complex Networks," Science, vol. 298, no. 5594, pp. 824-827, Oct. 2002.

[20] Ö. N. Yaveroğlu, N. Malod-Dognin, D. Davis, Z. Levnajic, V. Janjic, R. Karapandza, A. Stojmirovic, and N. Pržulj, "Revealing the Hidden Language of Complex Networks," Scientific Reports, vol. 4, p. 4547, Apr. 2014.

[21] H. Shakeri, P. Poggi-Corradini, N. Albin, and C. Scoglio, "Network clustering and community detection using modulus of families of loops," Physical Review E, vol. 95, no. 1, p. 012316, 2017.

[22] C. Klymko, D. Gleich, and T. G. Kolda, "Using triangles to improve community detection in directed networks," arXiv preprint arXiv:1404.5874, 2014.

[23] A. R. Benson, D. F. Gleich, and J. Leskovec, "Higher-order organization of complex networks," Science, vol. 353, no. 6295, pp. 163-166, 2016.

[24] — , "Tensor spectral clustering for partitioning higher-order network structures," in Proceedings of the 2015 SIAM International Conference on Data Mining. SIAM, 2015, pp. 118-126.

[25] R. Andersen, F. Chung, and K. Lang, "Local graph partitioning using pagerank vectors," in Foundations of Computer Science, 2006. FOCS'06. 47th Annual IEEE Symposium on. IEEE, 2006, pp. 475-486.

[26] P. Li and O. Milenkovic, "Inhomogeneous Hypergraph Clustering with Applications," in Advances in Neural Information Processing Systems, 2017, pp. 2308-2318.

[27] A. Lancichinetti, S. Fortunato, and J. Kertész, "Detecting the overlapping and hierarchical community structure in complex networks," New Journal of Physics, vol. 11, no. 3, p. 033015, Mar. 2009.

[28] C. Lee, F. Reid, A. McDaid, and N. Hurley, "Detecting highly overlapping community structure by greedy clique expansion," arXiv preprint arXiv: 1002.1827, 2010

[29] M. A. Javed, M. S. Younis, S. Latif, J. Qadir, and A. Baig, "Community detection in networks: A multidisciplinary review," Journal of Network and Computer Applications, vol. 108, pp. 87-111, Apr. 2018. 
[30] J. Xie, S. Kelley, and B. K. Szymanski, "Overlapping community detection in networks: The state-of-the-art and comparative study," ACM Computing Surveys, vol. 45, no. 4, pp. 1-35, Aug. 2013.

[31] D. Zhou, J. Huang, and B. Schölkopf, "Learning with Hypergraphs: Clustering, Classification, and Embedding," in Advances in Neural Information Processing Systems 19, B. Schölkopf, J. C. Platt, and T. Hoffman, Eds. MIT Press, 2007, pp. 1601-1608.

[32] P. Li and O. Milenkovic, "Submodular Hypergraphs: p-Laplacians, Cheeger Inequalities and Spectral Clustering," 2018, pp. 3020-3029.

[33] C. E. Tsourakakis, J. Pachocki, and M. Mitzenmacher, "Scalable Motifaware Graph Clustering," in Proceedings of the 26th International Conference on World Wide Web - WWW '17. Perth, Australia: ACM Press, 2017, pp. 1451-1460.

[34] B. Liao, X. Li, L. Cai, Z. Cao, and H. Chen, "A Hierarchical Clustering Method of Selecting Kernel SNP to Unify Informative SNP and Tag SNP," IEEE/ACM Trans. Comput. Biol. Bioinformatics, vol. 12, no. 1, pp. 113-122, Jan. 2015.

[35] A. Sarkar, Y. Ren, R. Elhesha, and T. Kahveci, "A new algorithm for counting independent motifs in probabilistic networks," IEEE/ACM Transactions on Computational Biology and Bioinformatics, pp. 1-1, 2018.

[36] S. Wernicke, "Efficient Detection of Network Motifs," IEEE/ACM Transactions on Computational Biology and Bioinformatics, vol. 3, no. 4, pp. 347-359, Oct. 2006.

[37] M. Latapy, "Main-memory triangle computations for very large (sparse (power-law)) graphs," Theoretical Computer Science, vol. 407, no. 1-3, pp. 458-473, 2008.

[38] N. H. Tran, K. P. Choi, and L. Zhang, "Counting motifs in the human interactome," Nature Communications, vol. 4, no. 1, p. 2241, Dec. 2013.

[39] J. Leskovec, "Stanford Large Network Dataset Collection," 2014.

[40] J. Leskovec and R. Sosič, "SNAP: A General-Purpose Network Analysis and Graph-Mining Library," ACM Transactions on Intelligent Systems and Technology, vol. 8, no. 1, pp. 1:1-1:20, Jul. 2016.

[41] A. Lancichinetti, S. Fortunato, and F. Radicchi, "Benchmark graphs for testing community detection algorithms," Physical review E, vol. 78, no. 4, p. 046110, 2008.

[42] M. Girvan and M. E. J. Newman, "Community structure in social and biological networks," Proceedings of the National Academy of Sciences, vol. 99 , no. 12, pp. 7821-7826, Jun. 2002

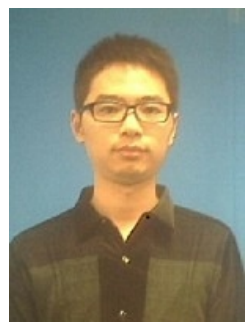

Wenhong Ma is currently working toward the Ph.D. degree with Hunan University, Changsha, China. His current research interests data mining,big data analysis, automotive cyber-physical systems, and embedded and cyber-physical systems.

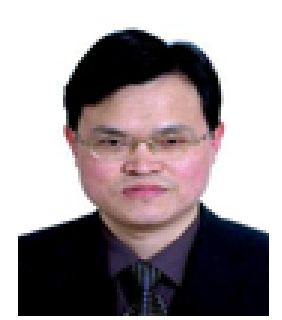

Lijun Cai received the Ph.D. degree in College of Computer Science and Electronic Engineering from Hunan University in 2007. He is currently a Professor at Hunan University. His research interests include bioinformatics, cloud computing, big data scheduling and management.

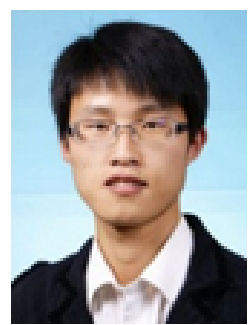

Tingqin He received the M.S. degree in College of Computer Science and Electronic Engineering, from Hunan University, Changsha, China. He is currently pursuing the Ph.D. degree in the College of Information Science and Engineering, Hunan University, Changsha, China. His research interests include date mining, cloud computing, big data analysis.

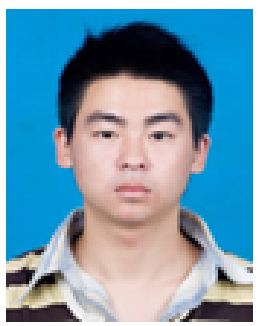

Lei Chen received the PhD degree from Hunan University in 2017 . He is currently a lecturer at Hunan University of Science and Technology. His research interests include date mining, web mining, graph mining, cloud computing, and big data scheduling and analysis.



Zehong Cao is a Lecturer (a.k.a. Assistant Professor) with Discipline of Information and Communication Technology (ICT), School of Technology, Environments and Design, College of Sciences and Engineering, University of Tasmania (UTAS), Hobart, Australia, and an Adjust Fellow with School of Computer Science, Faculty of Engineering and Information Technology, University of Technology Sydney (UTS), Australia. He received the dual PhD degree in Information Technology from UTS, and Electrical and Control Engineering from National Chiao Tung University (NCTU) in Taiwan. His research interests cover Fuzzy Sets and Systems, Fuzzy Neural Networks, Brain-Computer Interaction, Biosignal Processing, Game-based Machine Learning, and Data Mining. 